1989. 38,2

УДК $62-501.12$

Ю. НУРГЕС, К. КАСК, К. ЯАКСОО

\title{
ЦИФРОВЫЕ ФИЛЬТРЫ ЛАГЕРРА
}

\author{
(Представил Ю. Яаксоо)
}

\section{1. Введение}

Задача синтеза цифровых фильтров не вызывает принципиальных трудностей и весьма подробно исследована в области $z$-преобразования и непосредственно по весовым функциям. С точки зрения сжатия информации представляет интерес синтез фильтров в базисе ортогональных многочленов дискретного переменного [ $\left.{ }^{1}\right]$. Известна процедура синтеза нерекурсивного цифрового фильтра в базисе разностных многочленов Лагерра с использованием $z$-преобразования $\left[{ }^{2}\right]$.

В данной работе предлагается новый подход для синтеза фильтра по заданной весовой функции с применением разложения Лагерра дискретного переменного. Сначала выводится лагеррова модель линейной динамической системы в виде последовательности марковских параметров. Для вполне удовлетворяющей аппроксимации заданной весовой функции требуется ограниченное число первых марковских параметров лагерровой модели при подходящем выборе постоянной разложения. Отличительной чертой разностных многочленов Лагерра является простота сдвига их во временной области. Это свойство позволяет вычислить лагерровый спектр дискретного сигнала в реальном масштабе времени и вывести рекурсивный цифровой фильтр Лагерра.

\section{2. Разностные многочлены Лагерра}

Разностные многочлены Лагерра порядка $k$ можно привести к явному виду $\left[{ }^{3}\right]$ :

$$
\psi_{k}(t)=\sqrt{1-\xi^{2}} \sum_{j=0}^{k}(-1)^{k+j}\left(\begin{array}{l}
k \\
j
\end{array}\right)\left(\begin{array}{c}
t+k-j \\
k
\end{array}\right) \xi^{t+k-2 j},
$$

где $k, t=0,1,2, \ldots ; \xi \in(0,1),\left(\begin{array}{l}k \\ j\end{array}\right)$ - биномиальный коэффциент.

Вычисление многочленов $\psi_{k}(t)$ удобно проводить с помощью следующих рекуррентных формул:

$$
\begin{aligned}
& \psi_{0}(0)=\sqrt{1-\xi^{2}}, \\
& \psi_{0}(t+1)=\xi \psi_{0}(t), \\
& \psi_{k+1}(0)=-\xi \psi_{k}(0), \\
& \psi_{k}(t+1)=\xi \psi_{k}(t)+\left(1-\xi^{2}\right) \sum_{i=0}^{k-1}(-\xi)^{k-i-1} \psi_{i}(t),
\end{aligned}
$$


Так как многочлены $\psi_{k}(t)$ удовлетворяют условиям ортонормальности

$$
\sum_{t=0}^{\infty} \psi_{k}(t) \psi_{m}(t)=\delta_{k m}
$$

где $\delta_{k m}-$ символ Кронекера, то разложение дискретной функции $f(t), t=0,1, \ldots$ в ряд Фурье по многочленам $\psi_{k}(t)$ имеет простой вид:

$$
f(t)=\sum_{k=0}^{\infty} f_{k} \psi_{k}(t) .
$$

Здесь коэффициенты Фурье $f_{k}$ определяются соотношениями

$$
f_{k}=\sum_{t=0}^{\infty} f(t) \psi_{k}(t)
$$

По существу, задача синтеза фильтра Лагерра представляет собой задачу аппроксимации заданной весовой функции с помощью разложения Лагерра. Имеются два различных подхода для решения задачи.

Первый подход пригоден только для аппроксимации весовой функции специальных видов. Этот метод основан на сглаживающих свойствах преобразования Лагерра с конечным (небольшим) числом коэффициентов разложения. Подвергая дискретную функцию $u(t), t=$ $=0, \ldots, N$, последовательно преобразованиям (3) и (2) при $k=$ $=0, \ldots, M$, получим отфильтрованный сигнал $y(t), t=0, \ldots, N$, по формуле

$$
y(t)=\sum_{k=0}^{M} \psi_{k}(\tau) u_{k}(t), \quad 0 \leqslant \tau \leqslant N,
$$

где

$$
u_{k}(t)=\sum_{j=0}^{N} \psi_{k}(j) u(t-j), \quad t \geqslant N>M .
$$

Значит, весовая функция фильтра Лагерра определяется соотношением

$$
h(j)=\sum_{k=0}^{M} \psi_{k}(\tau) \psi_{k}(j), \quad j=0, \ldots, N
$$

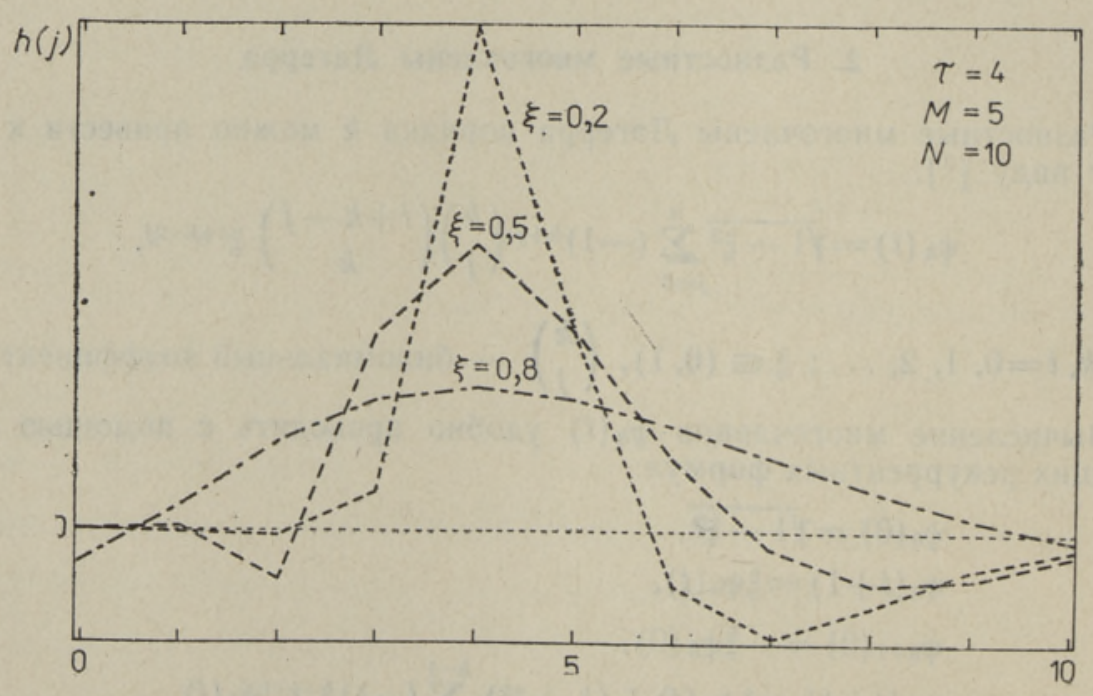

Рис. 1. Фильтрующие свойства преобразования Лаггера, 
Варьируя ร, $\tau$ и $M$, получим множество различных весовых функций $h(j, \xi, \tau, M)$ (рис. 1$)$.

\section{3. Нерекурсивный фильтр Лагерра}

Второй подход позволяет синтезировать фильтр с любой весовой функцией. Он заключается в переходе от временной модели (весовая функция) к лагерровой.

Рассмотрим динамическую систему (фильтр) в виде уравнений состояния

$$
\begin{aligned}
x(t+1) & =A x(t)+b u(t), \\
y(t) & =c^{\mathrm{T}} x(t), \quad t=0,1,2, \ldots,
\end{aligned}
$$

где $x(t)$ - вектор состояния, $u(t)-$ вход и $y(t)-$ выход фильтра. Разложив функции $x(t), u(t)$ и $y(t)$ по многочленам $\psi_{k}(t)$ и обозначив

$$
z_{k}=(I-\xi A) x_{k}-\xi b u_{k},
$$

получим уравнения состояния между коэффициентами разложения $\left[{ }^{3}\right]$

$$
\begin{aligned}
z_{k+1} & =\bar{A} z_{k}+\bar{b} u_{k}, \\
y_{k} & =\bar{c}^{\mathrm{T}} z_{k}+\bar{d} u_{k},
\end{aligned}
$$

причем

$$
\begin{aligned}
\bar{A} & =(A-\xi I)(I-\xi A)^{-1}, \\
\bar{b} & =\left(1-\xi^{2}\right)(I-\xi A)^{-1} b, \\
\bar{c}^{\mathrm{T}} & =c^{\mathrm{T}}(I-\xi A)^{-1}, \\
\bar{d} & =\xi c^{\mathrm{T}}(I-\xi A)^{-1} b, \\
z_{0} & =\sqrt{1-\xi^{2}} x(0) .
\end{aligned}
$$

Пусть теперь фильтр представлен весовой функцией $h(t), t=0,1, \ldots$,

$$
h(t)=\{y(t) \mid u(0)=1, x(0)=0, u(\tau)=0, \tau \neq 0\} .
$$

Найдем лагеррово разложение весовой функции

$$
h_{k}=\sum_{t=0}^{\infty} h(t) \psi_{k}(t), \quad k=0,1, \ldots,
$$

и соответствующего входного воздействия $u(t)$ (единичный импульс)

$$
u_{k}=(-\xi)^{k} \psi_{0}(0) \text {. }
$$

Связь между спектрами $u_{k}$ и $y_{k}$ можно выразить посредством лагерровой модели (4), (5). Так как $x(0)=0$, то из (6) $z_{0}=0$ и

$$
y_{k}=\bar{d} u_{k}+\sum_{j=0}^{k-1} \bar{c}^{\mathrm{T}} \bar{A}^{k-j-1} \bar{b} u_{j}
$$

или

$$
y_{k}=g_{0} u_{k}+\sum_{j=0}^{k-1} g_{k-j} u_{j},
$$

где марковские параметры лагерровой модели (т. н. лагеррова характеристика)

$$
\begin{aligned}
& g_{0}=\bar{d}, \\
& g_{i}=\bar{c}^{\mathrm{T}} \bar{A}^{i-1} \bar{b}, \quad i=1,2, \ldots .
\end{aligned}
$$

Лагеррову характеристику фильтра $g_{i}, i=0,1,2, \ldots$, , можно 
найти по разложению весовой функции фильтра $h_{k}$. Подставляя $u_{k}$ из (7) в (8), получим

$$
h_{k}=\psi_{0}(0)\left[(-\xi)^{k} g_{0}+\sum_{j=0}^{k-1}(-\xi)^{j} g_{j}\right],
$$

откуда

$$
g_{k}=\frac{1}{\psi_{0}(0)}\left(h_{k}+\xi h_{k-1}\right), \quad k=0,1, \ldots ; \quad g_{-1}=0 .
$$

Обработку данных нерекурсивным фильтром Лагерра можно осуществить следующим образом.

1. Выберем постоянную разложения $\xi, 0<\xi<1$.

2. По заданной весовой функции $h(t), t=0, \ldots, N$, найдем разложение Лагерра ее $h_{k}$ и по (9) - лагеррову характеристику фильтра $g_{k}, k=0, \ldots, M, M<N$.

3. По известной входной последовательности $u(t), t=0, \ldots, T$, найдем лагерровый спектр ее $u_{k}, k=0, \ldots, K, K>M, T>N$.

4. Вычислим по формуле (8) выходной спектр фильтра $y_{k}, k=$ $=0, \ldots, K$.

5. Посредством обратного преобразования (2) найдем последовательность отфильтрованных данных $y(t), t=0, \ldots, T$.

Результаты машинного эксперимента для трапециевидной весовой функции и постоянного входного сигнала приведены на рис. 2.

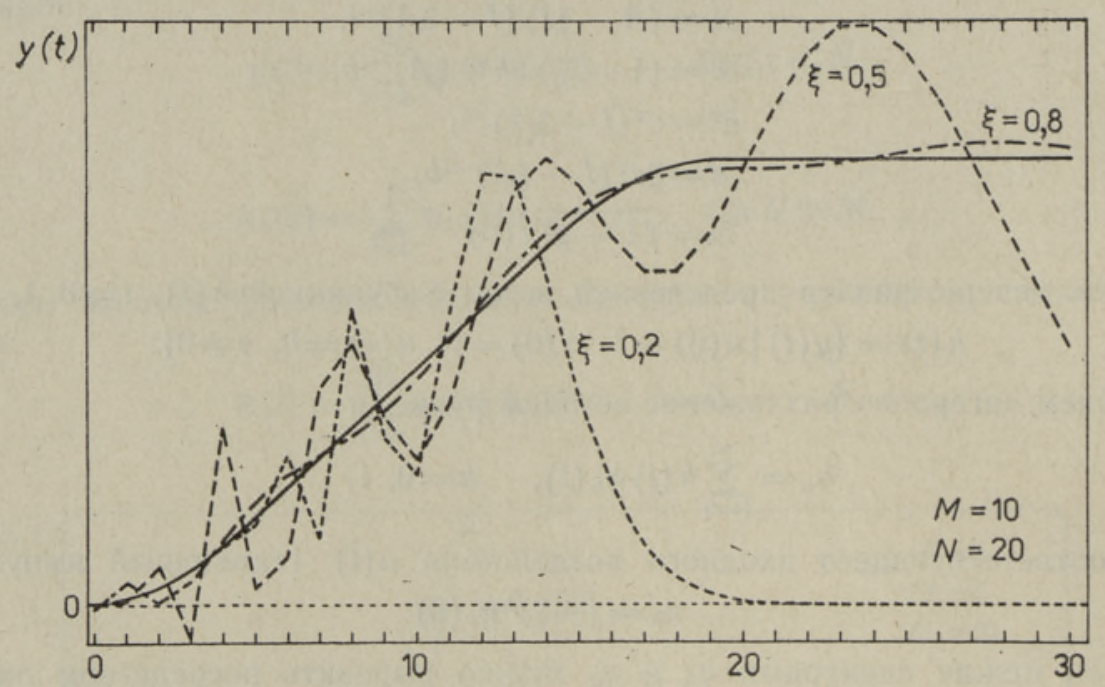

Рис. 2. Нерекурсивный фильтр Лагерра.

Определение оптимальной постоянной разложения сложная задача $\left[{ }^{2}\right]$. Отметим, что при больших $\xi$ лучше аппроксимируется конец, а при малых $\xi-$ начало весовой функции. Если полюсы желаемой характеристики фильтра известны, то для минимизации числа $M$ следует $\xi$ выбирать в центре тяжести полюсов фильтра $\left[{ }^{4}\right]$.

\section{4. Рекурсивный фильтр Лагерра}

Для рекурсивного фильтра входная последовательность $u(t), t=$ $=0,1, \ldots$, не фиксируется. Точнее, в каждый момент $t+1$ к известной последовательности $u(0), \ldots, u(t)$ прибавляется новый сигнал 
$u(t+1)$, влияние которого на лагерровый спектр $u_{k}, k=0, \ldots, M$, необходимо немедленно учесть. Учитывая свойства лагеррового преобразования (3), целесообразно менять направление временной последовательности.

Обозначим

$$
\bar{u}_{M}(t)=\left[\begin{array}{c}
u_{0}(t) \\
\vdots \\
u_{M}(t)
\end{array}\right]=\left[\begin{array}{ccc}
\psi_{0}(0) & \ldots & \psi_{0}(t) \\
\vdots & & \vdots \\
\psi_{M}(0) & \ldots & \psi_{M}(t)
\end{array}\right]\left[\begin{array}{c}
u(t) \\
\vdots \\
u(0)
\end{array}\right] .
$$

Тогда

$$
\bar{u}_{M}(t+1)=\left[\begin{array}{c}
\psi_{0}(0) \\
\vdots \\
\psi_{M}(0)
\end{array}\right] u(t+1)+\left[\begin{array}{ccc}
\psi_{0}(1) & \ldots & \psi_{0}(t+1) \\
\vdots & & \vdots \\
\psi_{M}(1) & \ldots & \psi_{M}(t+1)
\end{array}\right]\left[\begin{array}{c}
u(t) \\
\vdots \\
u(0)
\end{array}\right]
$$

или, учитывая свойство сдвига многочленов $\psi_{k}(t)$ во временной области (1), получим

$$
\bar{u}_{M}(t+1)=\Psi_{M}(0) u(t+1)+Q(\xi) \bar{u}_{M}(t),
$$

где

$$
\begin{aligned}
& \Psi_{M}(0)=\sqrt{1-\xi^{2}}\left[1,-\xi, \ldots,(-\xi)^{M}\right]^{\mathrm{T}} \text {, }
\end{aligned}
$$

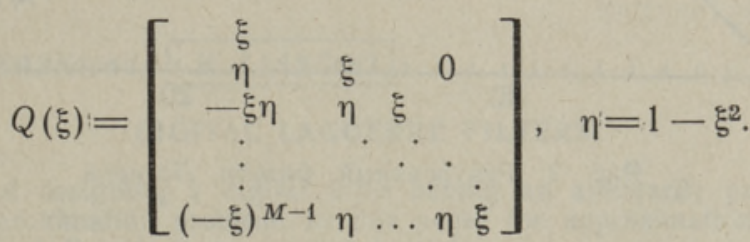

Пусть желаемое вход-выход соотношение фильтра следующее:

$$
y(t)=\sum_{j=0}^{N} h(j) u(t-j),
$$

где $h(j)$ - весовая функция фильтра. Посредством лагерровой характеристики $g_{k}$ найдем выходной спектр фильтра $y_{k}(t+1), k=0, \ldots, M$, в момент $t+1$

$$
y_{k}(t+1)=\sum_{i=0}^{M-k} g_{i} u_{k+i}(t+1)
$$

Выход ресурсивного фильтра Лагерра в момент $t+1$ определяется как функция входного спектра $u_{k}(t), k=0, \ldots, M$, в момент $t$ и входного сигнала $u(t+1)$

$$
y(t+1)=\alpha u(t+1)+\beta^{\mathrm{T}} \bar{u}_{M}(t),
$$

где

$$
\begin{gathered}
\alpha=\Psi_{M}^{\mathrm{T}}(0) G \Psi_{M}(0), \\
\beta^{\mathrm{T}}=\Psi_{{ }_{M}}^{\mathrm{T}}(0) G Q(\xi), \\
G=\left[\begin{array}{cccc}
g_{0} & g_{1} \cdots \cdots & g_{M} \\
& g_{0} & \ddots \\
0 & \searrow & g_{1} \\
& & g_{0}
\end{array}\right] .
\end{gathered}
$$

Процедура вычислений рекурсивного фильтра Лагерра следующая. 
1. Выберем постоянную разложения $\xi$, $0<\xi<1$, руководствуясь соображениями, приведенными в разделе 3 .

2. Вычислим скаляр $\alpha$ и вектор $\beta$ по формулам (13) и (14).

3. Лагерровый спектр входа $\widetilde{u}_{M}(t)$ вычислим рекурсивно по формуле $(11), \bar{u}_{M}(-1)=0$.

4. На каждом такте $t=0,1 . \ldots$ определим отфильтрованный $\mathrm{ch}$ нал $y(t)$ по формуле (12).

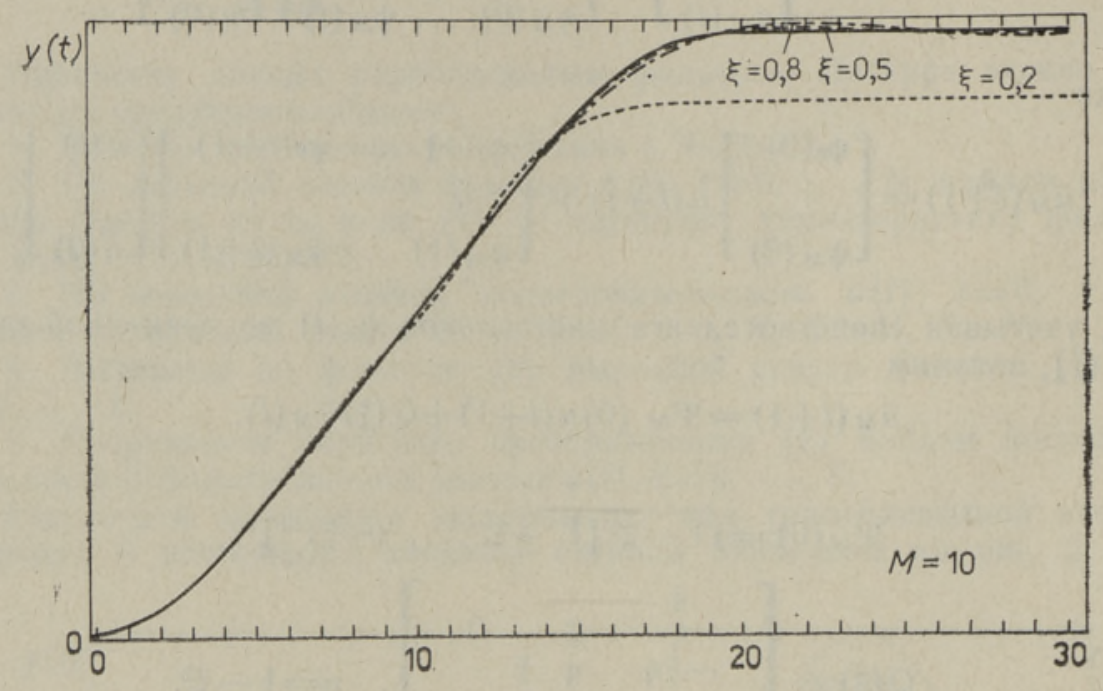

Рис. 3. Рекурсивный фильтр Лагерра.

Результаты машинного эксперимента для трапецеидальной весовой функции и постоянного входного сигнала приведены на рис. 3.

\section{5. Заключение}

Разработан метод для синтеза цифрового фильтра с применением разложения Лагерра дискретного переменного, который опирается на аппроксимации желаемой весовой функции фильтра лагерровой характеристикой. Основные этапы синтеза фильтра осуществлены в лагерровой области, т. е. вместо длинной весовой характеристики использована короткая лагеррова характеристика фильтра, вместо входных и выходных последовательностей - их лагерровы спектры.

Нерекурсивный фильтр Лагерра подходит только для пакетной обработки данных, а рекурсивный - для обработки в реальном масштабе времени. Машинные эксперименты показали, что лучшие результаты дает рекурсивный фильтр. Это и естественно, так как в рекурсивном фильтре происходит обновление информации на каждом такте, т. е. лагерровый спектр входа вычисляется рекурсивно в каждый момент времени.

Выбор коэффициента разложения $\xi$ зависит, в первую очередь, от желаемой динамики фильтра: для низкочастотного фильтра следует выбирать большой , а для высокочастотного - малый. Надо, однако, отметить, что оптимальный $\xi$ для рекурсивного фильтра, как правило, меньше оптимального $\xi$ для нерекурсивного фильтра. Причина опять же в обновлении информации на каждом такте, т. е. рекурсивный фильтр в большей мере учитывает начало весовой функции фильтра, 
1. Перов В. П. // Автоматика и телемеханика, 1976, № 10, 58-65; 1977, № 10, $65-73 ; 1978$, № 2, 53-61.

2. King, R. E., Paraskevopoulos, P. N. // Int. J. Circuit Theory and Appl., 1977, 5, № $1,81-91$.

3. Нургес Ю., Яаксоо Ю. // Изв. АН ЭССР. Физ. Матем., 1981, 30, № 3, 81-91.

4. Нуреес Ю. // Автоматика и телемеханика, 1987, № 3, 88-96.

\section{Институт кибернетики}

Академии наук Эстонской ССР

\section{Поступила в редакцию} $2 /$ II 1988

\section{O. NURGES, K. KASK, $\overline{\text { K. JAAKSOO }}$}

\section{DIGITAALSED LAGUERRE'I FILTRID}

On esitatud meetod digitaalse filtri sünteesiks diskreetsete Laguerre'i polünoomide baasil. Seosed sisend- ja vălljundsignaalide Laguerre'i spektrite vahel on esitatavad nn. Laguerre'i karakteristikuga, mis vôimaldab sünteesi ülesande üle viia Laguerre'i spektritele. Sobiva arendusteguri valikul võib pikad ajajadad asendada lühikeste Laguerre'i spektritega. Olenevalt töörežiimist vōib Laguerre'i filter olla rekursiivne (reaalaja töötlus) või mitterekursiivne (pakett-töötlus).

\section{O. NURGES, K. KASK and $K$.JAAKSOO}

\section{DIGITAL LAGUERRE FILTERS}

The problem of designing a digital filter having an arbitrarily prescribed impulse response is an approximation problem. In this paper the approximation is expressed in terms of orthonormal discrete Laguerre polynomials

$$
\psi_{k}(t)=\sqrt{1-\xi^{2}} \sum_{j=0}^{h}(-1)^{k+j}\left(\begin{array}{c}
k \\
j
\end{array}\right)\left(\begin{array}{c}
t+k-j \\
k
\end{array}\right) \xi^{t+k-2 j}, \quad \xi \in(0,1) .
$$

Due to the shift property of the polynomials $\psi_{k}(t)$, we obtain the recursive formula for an input Laguerre series

where

$$
\bar{u}_{M}(t+1)=\Psi_{M}(0) u(t+1)+Q(\xi) \bar{u}_{M}(t),
$$

$$
\bar{u}_{M}=\left[u_{0} \ldots u_{M}\right]^{\mathrm{T}}, \quad u_{k}=\sum_{\tau=0}^{\infty} u(t-\tau) \Psi_{k}(\tau) .
$$

The relation between input and output expansion coefficients $u_{k}$ and $y_{k}$ is as follows

$$
y_{k}=\sum_{j=0}^{h} g_{k-j} u_{j}
$$

where the Markov parameters of the Laguerre model $g_{k}$ can be expressed in terms of expansion coefficients $h_{k}$ of the desired impulse response $h(t), t=0,1,2, \ldots$

$$
g_{h}=\Psi_{0}^{-1}(0)\left(h_{k}+\xi h_{k-1}\right) \text {. }
$$

From (1) and (2) one can derive the digital Laguerre filter formula

where

$$
y(t+1)=\alpha u(t+1)+\beta^{\mathrm{T}} \bar{u}_{M}(t),
$$

$$
\begin{gathered}
\alpha=\Psi_{M}^{\mathrm{T}}(0) G \Psi_{M}(0), \\
\beta_{\tau}=\Psi_{M}^{\mathrm{T}}(0) G Q(\xi) .
\end{gathered}
$$

Some practical rules for choosing the discount factor $\xi$ are given. In general, for low-pass filter $\xi$ is great, and for high-pass filter $\xi$ is small, 